\title{
Comportamiento reológico no estacionario de emulsiones aceite en agua estabilizadas con un palmitato de sacarosa
}

\author{
Por P. Partal1, A. Guerrero2, M. Bejarano2 y C. Gallegos² \\ 1 Departamento de Ingeniería Química, Universidad de Huelva. \\ Escuela Politécnica Superior, La Rábida, 21819 Huelva. \\ 2 Departamento de Ingeniería Química, Universidad de Sevilla. \\ C/. P. García González, s/n. 41012 Sevilla
}

\section{RESUMEN}

\section{Comportamiento reológico no estacionario de emulsio- nes aceite en agua estabilizadas con un palmitato de saca-} rosa.

Se han estudiado emulsiones de calidad alimentaria aceite en agua estabilizadas por un palmitato de sacarosa de $H L B=15$. Dichas emulsiones se fabricaron a concentraciones comprendidas entre el 60 y el $80 \%(p / p)$ en peso de aceite vegetal con un $1-5 \%$ $(p / p)$ en peso del sucroéster empleado como emulsionante. Para llevar a cabo este estudio se realizaron ensayos de crecimiento del esfuerzo, flujo estacionario y cizalla oscilatoria. Todos los sistemas estudiados presentan un comportamiento tixotrópico en flujo transitorio. Bajo determinadas condiciones, la cizalla puede hacer que el esfuerzo vuelva a aumentar con el tiempo tras haber alcanzado un valor pseudoestacionario. Este hecho se ha relacionado con una redistribución de tamaños de gota como consecuencia de la cizalla. Como resultado, la cizalla provoca una intensa destrucción estructural en los sitemas estudiados, no pudiéndose ajustar la ecuación de Arrhenius a todo el intervalo de temperaturas estudiado.

PALABRAS-CLAVE: Emulsión - Emulsionante - Flujo transitorio - Palmitato de sacarosa - Reología.

\section{SUMMARY}

Non-steady rheological behavior of oil-in-water emulsions stabilized by a sucrose palmitate.

Non-steady flow properties of concentrated oil in water emulsions containing oil ( $60-80 \%$ wt.), water and sucrose palmitate (1-5\% wt.) of high hydrophilic-lypophilic balance were investigated. Stress growth, steady flow and linear viscoelastic measurements were carried out for this study. The emulsions studied exhibited a thixotropic behavior showing a very rapid stress overshoot. The stress decay was fitted to a Figoni-Shoemaker model using two exponentials. An stress undershoot was also produced under certain conditions. Shear brought about strong structural changes, such as droplet size redistribution at high oil content and temperature. Thus, the steady state viscosity does not fit an Arrhenius type equation with temperature. A shear thinning behavior was found for the steady state viscosity and the peak viscosity.

KEY-WORDS: Emulsifier - Emulsion - Rheology - Sucrose palmitate - Transient flow.

\section{INTRODUCCION}

Los ésteres de sacarosa son tensioactivos no iónicos que pueden obtenerse a partir de materias primas naturales, abundantes y de bajo coste, tales como aceites vegetales y sacarosa. Gracias a estas características, los ésteres de sacarosa encuentran aplicación como emulsionantes tanto en la industria alimentaria como en las industrias cosmética y farmacéutica (Walker, 1984; Yuki et al., 1990; Desai, 1990).

Las diferentes posiciones de esterificación de la molécula de sacarosa convierten a sus ésteres en una familia de emulsionantes muy versátil que cubre un amplio intervalo de valores del balance hidrófilo-lipófilo (HLB). Naturalmente, las propiedades de los ésteres de sacarosa dependen en gran medida del valor del HLB, siendo los valores altos los más adecuados para la estabilización de emulsiones aceite en agua estables (Akoh y Nwosu, 1992).

La estabilidad de la emulsión final (desde el punto de vista cinético) mejora considerablemente por la adición de un agente emulsionante. En emulsiones o/w el papel del emulsionante consiste en su absorción en la interfase agua-aceite formando una barrera protectora en torno a las gotas de aceite, que incrementa las interacciones entre gotas y que conduce a una mejora de la estabilidad frente a la coalescencia (Dickinson, 1994).

La estabilidad y la viscoelasticidad de la emulsión están muy relacionadas entre sí, ambas muy influidas por los parámetros estructurales, sobre todo la concentración de la fase dispersa y las interacciones entre las gotas. No obstante, otras variables tales como tamaño y polidispersión de las gotas, naturaleza y propiedades de la fase continua y de la interfase, etc., también pueden presentar una influencia considerable sobre la estabilidad y comportamiento reológico (Rahalkar, 1992; Tadros, 1993).

La estabilidad coloidal y el comportamiento reológico de emulsiones concentradas dependen conside- 
rablemente del balance entre las fuerzas atractivas, corrientemente las fuerzas de van der Waals, de largo alcance, y las fuerzas repulsivas. Estas últimas pueden ser de naturaleza electrostática, en cuyo caso dependen del espesor de la doble capa que rodea a las gotas, o interacciones estéricas que tienen lugar cuando una capa de emulsionante o macromolecular rodea a las gotas. Si las fuerzas de van der Waals son débiles, contribuyen a favorecer la formación de estructuras reversibles tipo entramado y en consecuencia pueden incrementar la estabilidad. Sin embargo, si éstas son fuertes pueden producir desestabilización de la emulsión (floculación y coalescencia). Valores altos de potenciales electrostáticos y estéricos van acompañados de considerables incrementos de estabilidad y viscoelasticidad del sistema disperso (como ejemplo puede darse el caso de la mayonesa) (Rahalkar, 1992).

La cizalla puede modificar sustancialmente el balance de fuerzas y por tanto la estabilidad y viscoelasticidad de la emulsión, dependiendo de la magnitud y del tiempo de la cizalla aplicada. Estas modificaciones pueden ser reversibles, mediante un proceso de floculación-defloculación o irreversibles como es el caso de la coalescencia inducida por la cizalla o la rotura de gotas (Darby, 1984; Dickinson, 1994).

En consecuencia el conocimiento del comportamiento reológico, abarcando desde ensayos de pequeña deformación (próximos al estado de la emulsión sin perturbación) hasta ensayos con cizalla elevada (próximos al sistema completamente defloculado), es de gran importancia para caracterizar la estructura y estabilidad de las emulsiones y mejorar la comprensión del proceso de emulsificación durante el cual es típica la existencia de fuerzas de cizalla de elevada cuantía.

Las propiedades reológicas dependientes del tiempo reflejan la naturaleza de un sistema estructurado y pueden deberse a viscoelasticidad, cambios estructurales o a ambos efectos (Cheng y Evans, 1965). La viscoelasticidad se caracteriza por una respuesta no instantánea a un esfuerzo aplicado al sistema, mientras que un cambio estructural se caracteriza por una respuesta instantánea a una velocidad aplicada.

En este trabajo se aborda el estudio de la dependencia con el tiempo de las propiedades reológicas desde el punto de vista de destrucción estructural, analizando la influencia de la velocidad de cizalla, de las concentraciones de sucroéster y aceite y de la temperatura.

\section{EXPERIMENTAL}

Las emulsiones aceite en agua se han preparado utilizando un palmitato de sacarosa, con un balance hidrófilo-lipófilo (HLB) de 15, suministrado por Mitsu-
bishi-Kasei Food Corporation (Tokio, Japón). El aceite de girasol utilizado fue adquirido en el mercado.

El procesado de las emulsiones se realizó de la manera siguiente: se disolvió el éster de sacarosa en agua destilada a $55^{\circ} \mathrm{C}$ y a continuacón y sobre esta disolución, se añadió el aceite a temperatura ambiente, a una velocidad de $70 \mathrm{~g} / \mathrm{min}$. Durante todo el proceso se sometió la mezcla a agitación mecánica empleando un agitador de turbina «Ultra Turrax» T-50 (Ika, Alemania). Las emulsiones se han preparado a 5000 rpm empleando tiempos de emulsificación de 3,5 minutos.

El intervalo de concentraciones estudiado fue $1-5 \%(\mathrm{p} / \mathrm{p})$ de éster de sacarosa (SE), manteniendo constante la concentración de aceite $(70 \% \mathrm{p} / \mathrm{p})$. En el caso del aceite $(\mathrm{O})$ el intervalo de concentraciones estudiado se ha variado entre el 60 y $80 \%(\mathrm{p} / \mathrm{p})$ manteniendo constante la concentración de sucroéster (3\% p/p). Todas las emulsiones fueron almacenadas a $5^{\circ} \mathrm{C}$. El intervalo de temperaturas estudiado fue de 5 a $35^{\circ} \mathrm{C}$.

Los ensayos de crecimiento del esfuerzo y de flujo estacionario se han llevado a cabo en un viscosímetro rotatorio Haake RV-100-CV-100 y RV20-CV20N, empleándose sistemas sensores del tipo de cilindros coaxiales, ME-16 y ME-31 $\left(R_{\mathrm{e}} / \mathrm{R}_{\mathrm{i}}=1,037\right)$. Los ensayos de flujo transitorio se efectuaron a velocidades de cizalla de 0,$6 ; 1,2 ; 1,8 ; 3 ; 6$ y $18 \mathrm{~s}^{-1}$. Los resultados de flujo estacionario se obtuvieron tras cizallar las muestras a $300 \mathrm{~s}^{-1}$ durante 10 minutos, tras lo cual se obtuvieron las curvas en el intervalo de 300 a $0,1 \mathrm{~s}^{-1}$. Los ensayos de cizalla oscilatoria se llevaron a cabo en un reómetro Haake RheoStress RS100, utilizando un sistema sensor del tipo cono-placa de $35 \mathrm{~mm}$ de diámetro y $4^{\circ}$ de ángulo. Todas las muestras fueron sometidas a la misma historia termorreológica.

Las distribuciones de tamaños de gotas de las emulsiones se obtuvieron en un analizador de tamaño de partícula Coulter Counter modelo $Z_{B}$ (Coulter Electronics Ltd.), utilizando un tubo de diámetro de apertura de $100 \mu \mathrm{m}$.

\section{ANÁLISIS Y DISCUSIÓN DE LOS RESULTADOS}

\subsection{Variación del esfuerzo de cizalla con el tiempo}

En las figuras 1 y 2 se representan los resultados obtenidos en las experiencias de variación del esfuerzo en función del tiempo, para cada una de las velocidades, concentraciones y temperaturas estudiadas. Como se observa en dichas figuras el comportamiento transitorio de estas emulsiones es similar. A saber, un crecimiento del esfuerzo muy rápido hasta alcanzar un valor máximo (sobreesfuerzo), seguido de una caída que se va amortiguando paulatinamente y que tiende a alcanzar un valor constante. Si la velocidad de cizalla es suficientemente alta, puede aparecer 
además un valor mínimo del esfuerzo tras el cual se observa un aumento de éste con el tiempo, como puede apreciarse en la figura 3 para la concentración $3 \%$ de sucroéster y $80 \%$ de aceite a $25^{\circ} \mathrm{C}$. Esta situación se encuentra con relativa frecuencia en la bibliografía de emulsiones concentradas (Guerrero et al., 1992; Muñoz et al., 1994). En la figura 3 se puede observar que el mínimo del esfuerzo aparece a un valor aproximadamente constante del producto $\dot{\gamma} \cdot t$. Por tanto, este efecto puede aparecer también a $\dot{\gamma}$ moderada siempre que el tiempo de experimentación sea prolongado.

La aparición de un mínimo del esfuerzo y posterior tendencia a alcanzar un segundo máximo, se ha observado que depende también de la temperatura (figura 4). Así se ha encontrado una mayor tendencia a la aparición del mínimo cuando aumenta la tempe- ratura, siendo más fácil que aparezca a alta concentración de aceite (figura 2). Un aumento del esfuerzo con el tiempo debe estar relacionado con un incremento de las interacciones entre gotas. Para determinar la causa de dicho aumento, se analizaron las distribuciones de tamaños de gota antes y después de la cizalla a $35^{\circ} \mathrm{C}$. En la figura 4 se presentan las distribuciones de tamaños, como frecuencia acumulativa y como porcentaje de volumen, de la muestra antes y después de ser cizallada. Se puede observar que tras la cizalla la emulsión presenta una distribución de tamaños más estrecha y con tendencia a un menor diámetro medio. Para comprobar esto, los datos experimentales de distribución acumulada de tamaños se han ajustado a la siguiente ecuación (Partal et al., 1994):
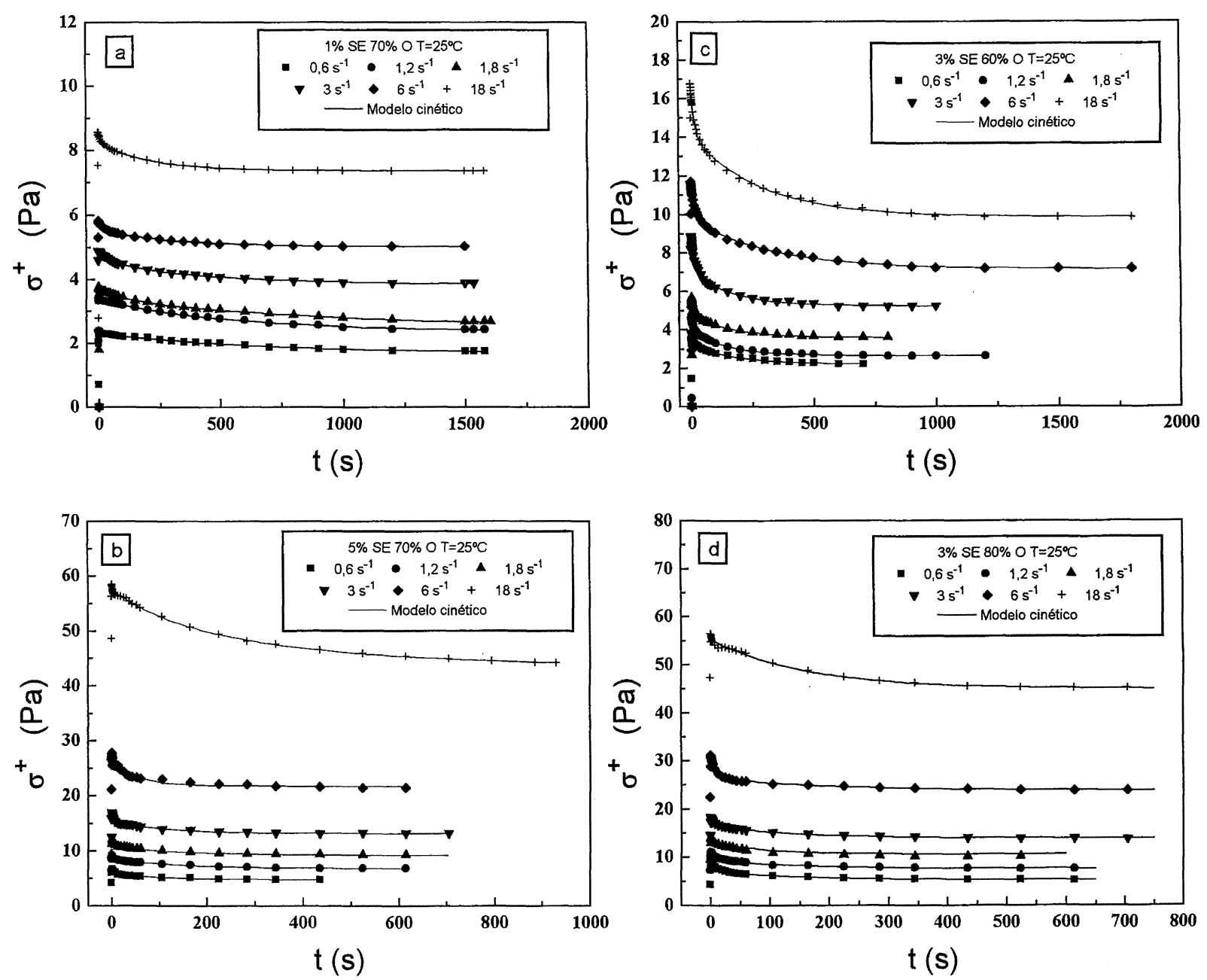

Figura 1

Flujo transitorio a $25^{\circ} \mathrm{C}$ en emulsiones con diferente concentración: a) $1 \%$ de sucroéster y $70 \%$ de aceite; b) $5 \%$ de sucroéster y $70 \%$ de aceite; c) $3 \%$ de sucroéster y $60 \%$ de aceite; d) $3 \%$ de sucroéster y $80 \%$ de aceite. 


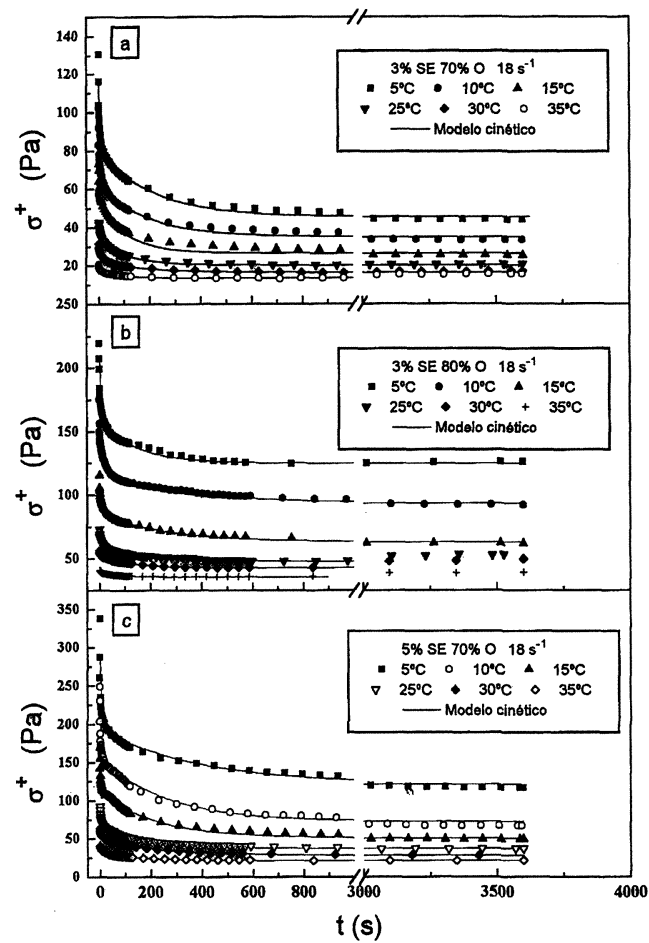

Figura 2

Flujo transitorio a $18 \mathrm{~s}^{-1}$ en emulsiones con diferente concentración: a) 3\% de sucroéster y $70 \%$ de aceite; b) $3 \%$ de sucroéster y $80 \%$ de aceite; c) $5 \%$ de sucroéster y $70 \%$ de aceite.

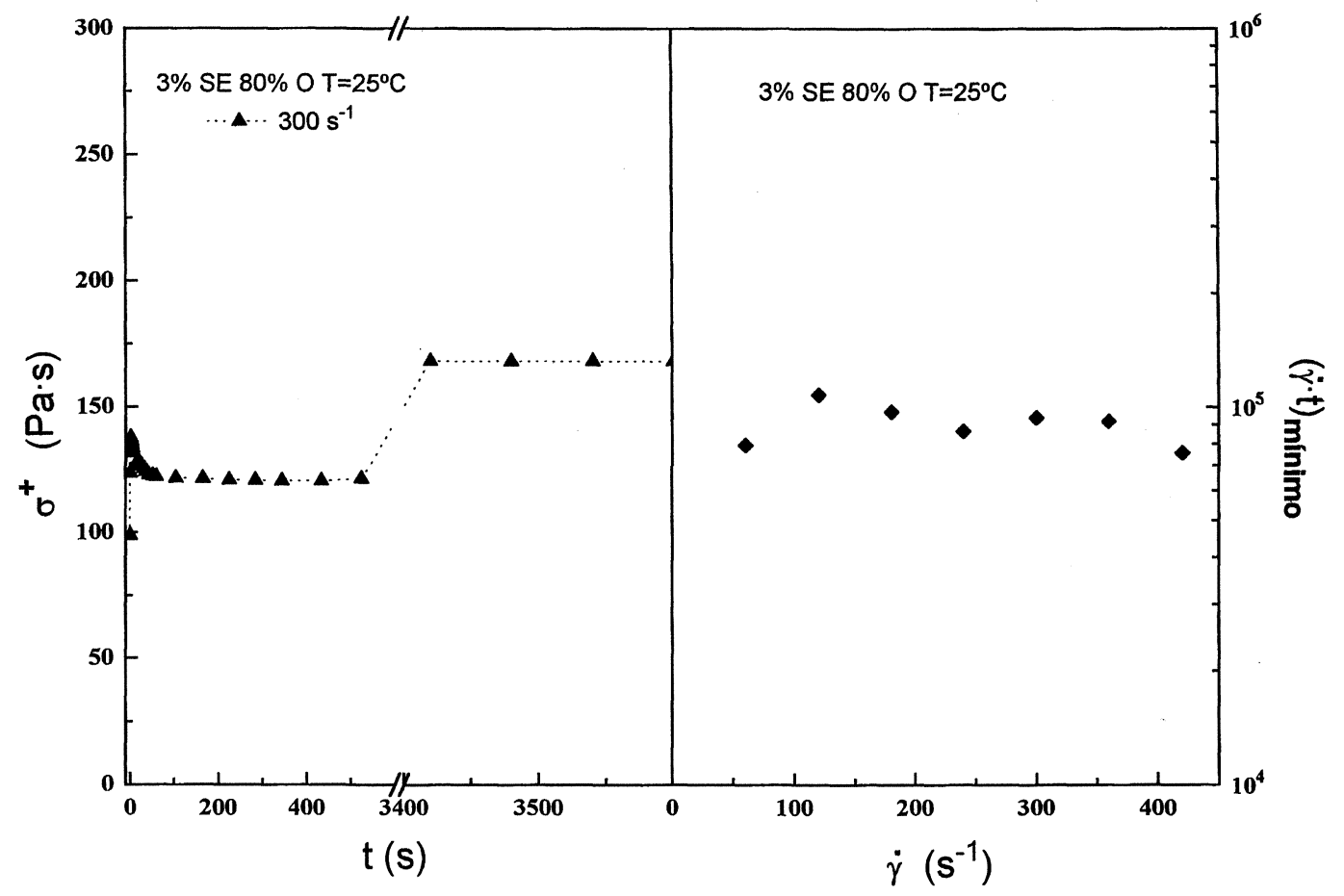

Figura 3

Curva de flujo transitorio para una emulsión con un $80 \%$ de aceite a $300 \mathrm{~s}^{-1}$ y $25^{\circ} \mathrm{C}$.

Valores de la "deformación» aplicada en el mínimo del esfuerzo a $25^{\circ} \mathrm{C}$. 


$$
f_{c}=\frac{1}{1+\left(d_{1 / 2} / d\right)^{b}}
$$

donde $d_{1 / 2}$ es la mediana de la distribución de tamaños y b es un parámetro inversamente proporcional a la dispersión de los mismos. Los resultados del ajuste (Tabla I) muestran que tras la cizalla el parámetro $\mathrm{b}$ aumenta (distribución más estrecha) y el diámetro de Sauter $\left(\mathrm{d}_{\mathrm{sv}}\right)$ disminuye (aumenta la superficie específica). El mismo efecto se ha encontrado en emulsiones estabilizadas con un polietilenglicol nonilfenil éter con 10 grupos etoxilados (Sánchez, 1997), incluyendo la aparición del mínimo del esfuerzo y la redistribución de tamaños de gota a largos tiempos de cizalla. Esta redistribución de tamaños estaría favorecida por un proceso inicial de coalescencia inducida por cizalla, como se ha detectado en emulsiones estabilizadas por proteínas de huevo (Madiedo et al., 1997), seguido de un proceso de reducción del tamaño de gota por la acción de las fuerzas de cizalla a lo largo del tiempo. La coalescencia conduce a mayores tamaños de gota que requieren menores valores de cizalla para su rotura. Emulsiones concentradas estabilizadas con un éster de sacarosa de alto HLB también muestran el mismo efecto de coalescencia y reducción de tamaños.

Estos efectos podrían ser la causa del incremento de las interacciones entre gotas $y$, por tanto, del aumento del esfuerzo tras el mínimo. La aparición de este mínimo estaría favorecida por las condiciones extremas de temperatura, concentración y velocidad de cizalla.
Tabla I

Influencia de la cizalla sobre la distribución de tamaños de emulsiones que contienen un $80 \%$ de aceite

\begin{tabular}{lccc}
\hline Muestra & $\mathbf{d}_{\mathbf{1 / 2}}(\boldsymbol{\mu m})$ & $\mathbf{b}$ & $\mathbf{d}_{\mathbf{s v}}(\boldsymbol{\mu m})$ \\
\hline Sin cizallar & 2,4 & 3,4 & 5,5 \\
Cizallada & 2,3 & 4,0 & 4,5 \\
\hline
\end{tabular}

\subsection{Influencia de la velocidad de cizalla y de la temperatura}

En todos los casos se ha encontrado que tanto el esfuerzo en el máximo como en el equilibrio dependen de la velocidad de cizalla. En la figura 5 se observa que estas emulsiones presentan un comportamiento pseudoplástico en el intervalo de $\dot{\gamma}$ estudiado, es decir, se produce una disminución de la viscosidad con la velocidad de cizalla. Este comportamiento puede ser descrito por el modelo de Ostwald-de Waele (figura 5):

$$
\eta=k \dot{\gamma}^{n-1}
$$

donde $\mathrm{k}$ y $\mathrm{n}$ son los índices de consistencia y de flujo respectivamente. Los valores de ambos parámetros del modelo se presentan en la Tabla II.

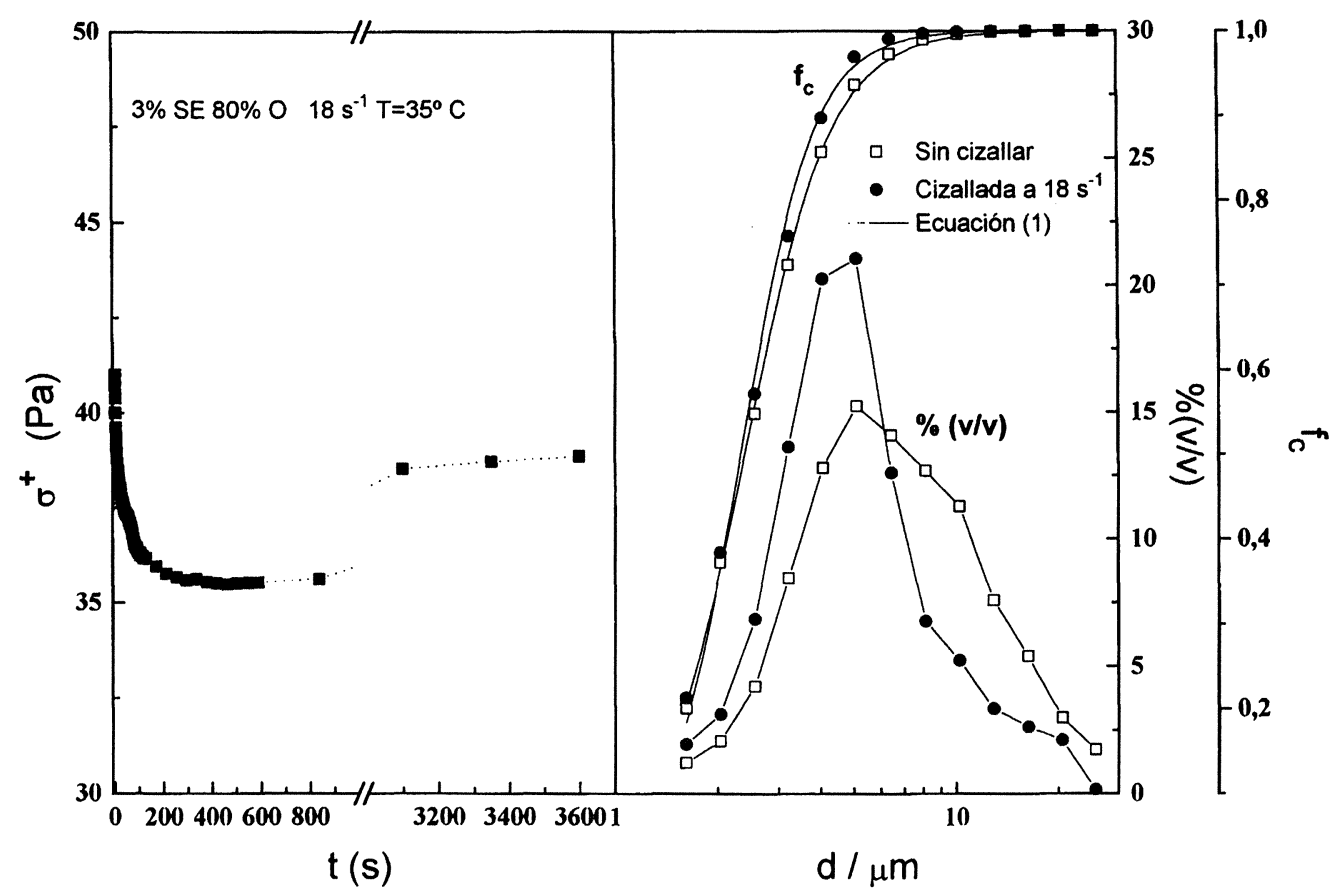

Figura 4

Curva de flujo transitorio para una emulsión con un $80 \%$ de aceite a $18 \mathrm{~s}^{-1}$ y $35^{\circ} \mathrm{C}$. Distribución de tamaños de gota antes y después de la cizalla. 


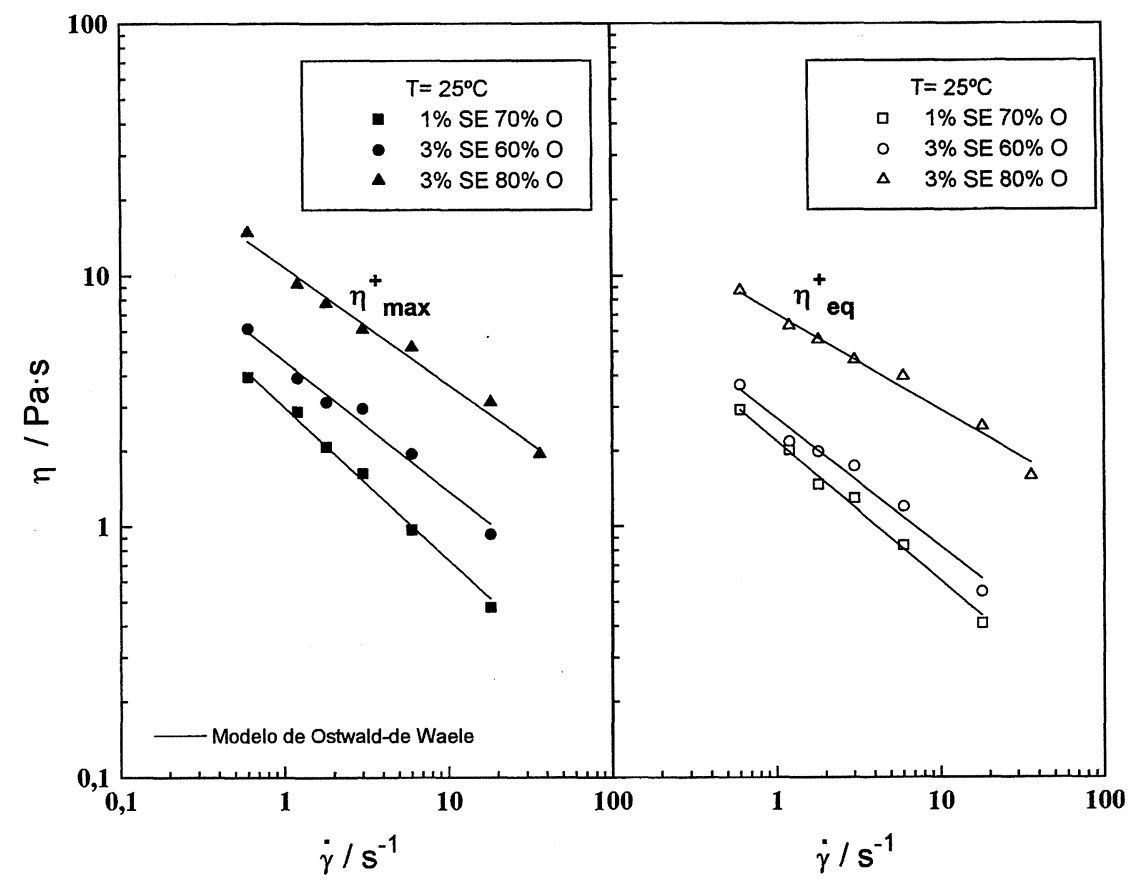

Figura 5

Variación de la viscosidad en el máximo y en el equilibrio con la velocidad de cizalla a $25^{\circ} \mathrm{C}$

Tabla II

Parámetros del modelo Ostwald-de Waele en emulsiones con diferente concentración, obtenidos a $25^{\circ} \mathrm{C}$

\begin{tabular}{|c|c|c|c|c|c|}
\hline \multirow[b]{2}{*}{$\%$ SE } & \multirow[b]{2}{*}{$\%$} & \multicolumn{2}{|c|}{$\eta^{+}{ }_{\max }$} & \multicolumn{2}{|c|}{$\eta^{+}{ }_{\mathrm{eq}}$} \\
\hline & & $k$ (Pa.sn) & $\mathbf{n}$ & $k\left(P a \cdot s^{n}\right)$ & $\mathbf{n}$ \\
\hline $1 \%$ & $70 \%$ & 3,0 & 0,390 & 2,2 & 0,443 \\
\hline $3 \%$ & $70 \%$ & 5,6 & 0,583 & 3,9 & 0,556 \\
\hline $5 \%$ & $70 \%$ & 8,6 & 0,631 & 6,4 & 0,660 \\
\hline $3 \%$ & $60 \%$ & 4,6 & 0,480 & 2,7 & 0,487 \\
\hline $3 \%$ & $80 \%$ & 10,8 & 0,532 & 7,1 & 0,618 \\
\hline
\end{tabular}

La influencia de la temperatura sobre la viscosidad en el máximo y en el equilibrio se presenta en las figuras 6 y 7 . Se observa un descenso de ambos parámetros con la temperatura que puede ajustarse a una ecuación de tipo Arrhenius:

$$
\eta_{\max }^{+}=A \exp \left(\frac{E_{a}}{R T}\right)
$$

$$
\eta_{\mathrm{eq}}^{+}=A \exp \left(\frac{E_{\mathrm{a}}}{R T}\right)
$$

donde $E_{a}$ es la energía de activación. En el caso de la viscosidad en el máximo su variación con la temperatura puede ser descrita, en todo el intervalo, por dicha ecuación y los resultados de las energías de activación se presentan en la Tabla III. Sin embargo, para la viscosidad en estado estacionario se pueden observar 
dos zonas de pendientes diferentes, una a temperatura alta y otra a baja. El efecto de la temperatura sobre la viscosidad de la emulsión a temperatura baja es mayor, por lo que los valores de la energía de activación son más pequeños a alta temperatura (Tabla III). Este comportamiento anómalo se puede explicar teniendo en cuenta que al estado estacionario se llega tras un tiempo de cizalla, a diferencia del máximo que se alcanza casi instantáneamente. Como previamente se ha demostrado, bajo condiciones intensas de cizalla o temperatura se puede observar un aumento de la viscosidad con el tiempo de cizalla, que está relacio- nado con una redistribución de tamaños de gota en la emulsión. Este aumento de viscosidad puede ser la causa de la menor susceptibilidad de la emulsión a alta temperatura. Así, los resultados parecen indicar (figura 7) que para temperaturas inferiores a $15^{\circ} \mathrm{C}$ se produce una destrucción estructural donde la redistribución de tamaños no es apreciable, obteniéndose valores de $E_{a}$ más altos. Por el contrario, en el intervalo de temperaturas de 25 a $35^{\circ} \mathrm{C}$ la redistribución de tamaños parece ser más intensa, con lo que se obtienen menores valores de energía de activación.

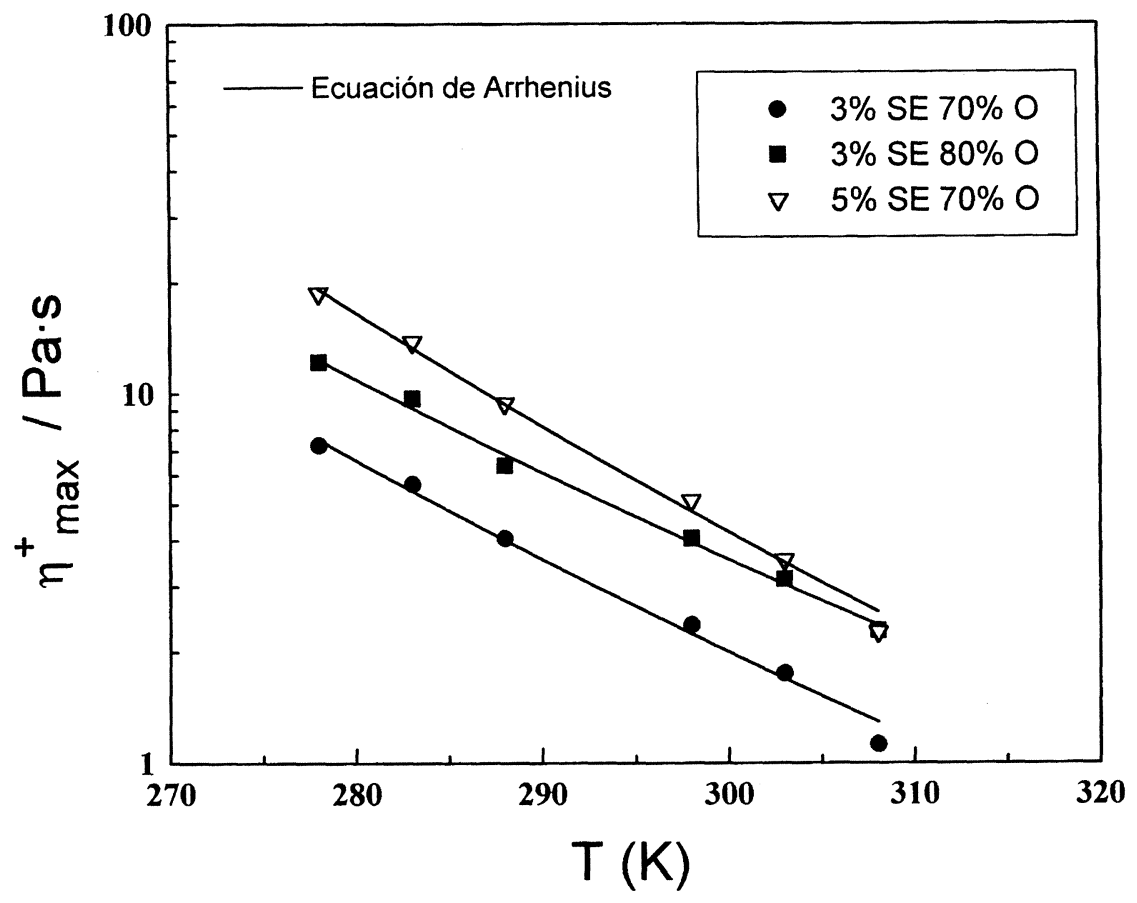

Figura 6

Variación con la temperatura de la viscosidad en el máximo obtenida a $18 \mathrm{~s}^{-1}$

Tabla III

Energías de activación obtenidas en el intervalo de temperaturas indicado para emulsiones con diferente cocentración

\begin{tabular}{|c|c|c|c|c|}
\hline$\%$ SE & $\%$ & $\begin{array}{c}\mathrm{E}_{\mathrm{a}}\left(\eta^{+}{ }_{\max }\right)(\mathrm{kJ} / \mathrm{mol}) \\
5-35^{\circ} \mathrm{C}\end{array}$ & $\begin{array}{c}\mathrm{E}_{\mathrm{a}}\left(\eta^{+}{ }_{\mathrm{eq}}\right)(\mathrm{kJ} / \mathrm{mol}) \\
5-15^{\circ} \mathrm{C}\end{array}$ & $\begin{array}{c}\mathrm{E}_{\mathrm{a}}\left(\eta^{+}{ }_{\text {eq }}\right)(\mathrm{kJ} / \mathrm{mol}) \\
25-35^{\circ} \mathrm{C}\end{array}$ \\
\hline $3 \%$ & $70 \%$ & 41,8 & 37,1 & 21,8 \\
\hline $5 \%$ & $70 \%$ & 47,9 & 59,7 & 40,4 \\
\hline $3 \%$ & $80 \%$ & 39,3 & 46,9 & 22,9 \\
\hline
\end{tabular}




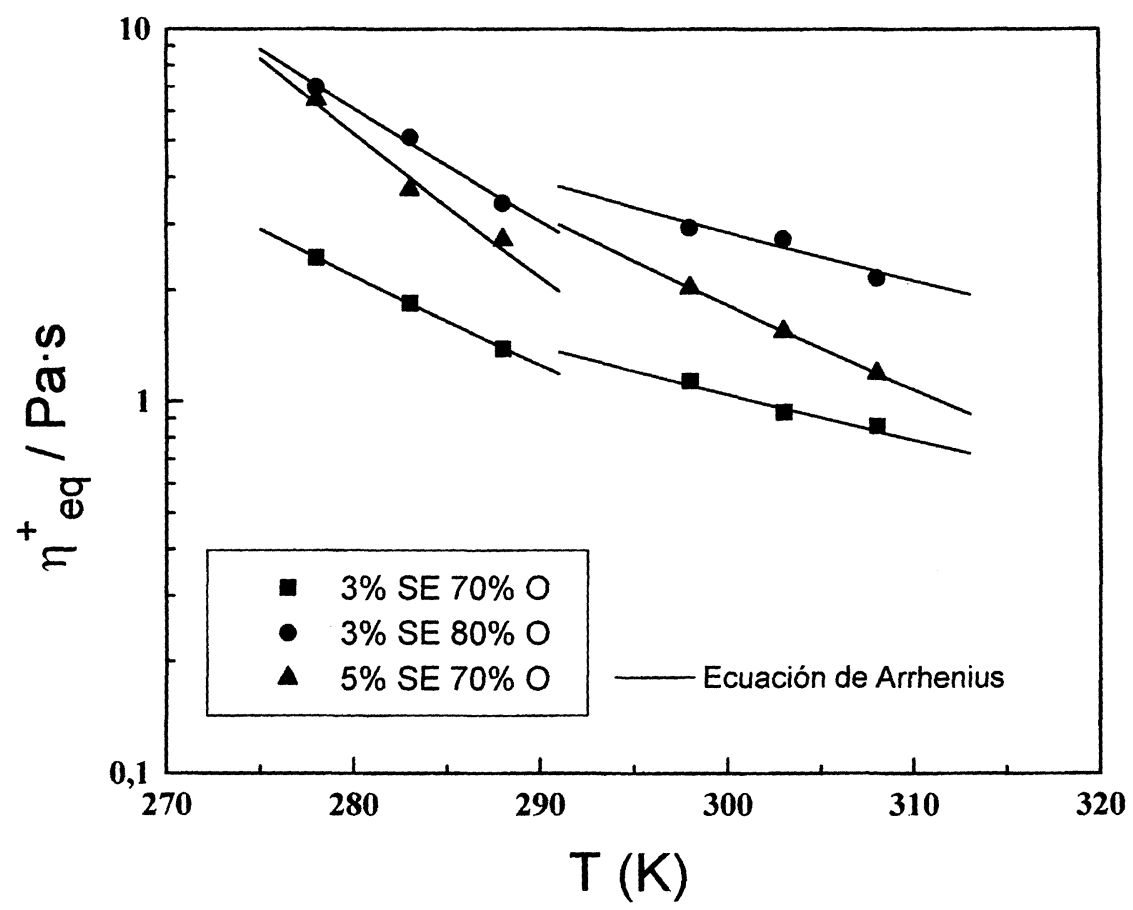

Figura 7

Variación con la temperatura de la viscosidad en el equilibrio obtenida a $18 \mathrm{~s}^{-1}$

\subsection{Estudio de la destrucción estructural}

El estudio de la destrucción estructural que se produce en estas emulsiones como consecuencia de la cizalla se ha realizado analizando la cantidad de sobreesfuerzo, el porcentaje de reodestrucción y comparando los resultados obtenidos en viscoelasticidad lineal con los obtenidos en viscoelasticidad no lineal.

\subsubsection{Comparación entre ensayos en cizalla oscilatoria, transitoria y estacionaria}

En disoluciones de polímeros, Cox y Merz (1958) establecieron una regla empírica según la cual la viscosidad compleja a una determinada frecuencia es igual a la viscosidad en cizalla estacionaria a una velocidad de cizalla igual a la citada frecuencia:

$$
\eta^{*}(\omega)=\eta(\dot{\gamma})_{\dot{\gamma}=\omega}
$$

Las viscosidades compleja, estacionaria y transitoria tanto en el máximo como en el equilibrio, se representan frente a la frecuencia y la velocidad de cizalla en la figura 8. En todos los casos se observa un comportamiento de tipo pseudoplástico respecto a la velocidad de cizalla, presentando además la viscosidad estacionaria una zona newtoniana a baja velocidad de cizalla.

Para todas las concentraciones estudiadas se ha encontrado que la regla de Cox-Merz no se cumple, encontrándose grandes diferencias entre los resultados obtenidos en viscoeslaticidad lineal y los encontrados en viscoeslaticidad no lineal. No se han encontrado diferencias importantes entre los valores de viscosidad en el equilibrio y los de viscosidad en flujo estacionario, las mayores diferencias se han obtenido a baja velocidad de cizalla, como consecuencia de la mayor tendencia a alcanzar una viscosidad newtoniana a baja velocidad de cizalla en los ensayos de cizalla en flujo estacionario, lo cual se puede deber a que las muestras fueron previamente cizalladas a $300 \mathrm{~s}^{-1}$.

De los resultados se puede deducir que la cizalla produce una fuerte destrucción estructural en la emulsión (figura 8), destrucción que es muy importante incluso antes de alcanzar el máximo del esfuerzo. Esta destrucción se puede atribuir a procesos reversibles (defloculación) e irreversibles, como otros autores han descrito previamente en emulsiones alimentarias (Figoni y Shoemaker, 1983; Gallegos et al., 1988; Alfaro, 1993); sin embargo las diferencias obtenidas son mucho mayores que las encontradas en otras emulsiones alimentarias comerciales (Berjano et al., 1991). Por tanto, el alto grado de estructuración que el sistema alcanza en estado de reposo (floculación) resulta extraordinariamente frágil a la cizalla. Por otro lado, la curva de la viscosidad en el máximo estará probablemente muy influida por la inercia del aparato, es decir, el sobreesfuerzo deberá aparecer a tiempos más cortos de los que realmente se detectan. 


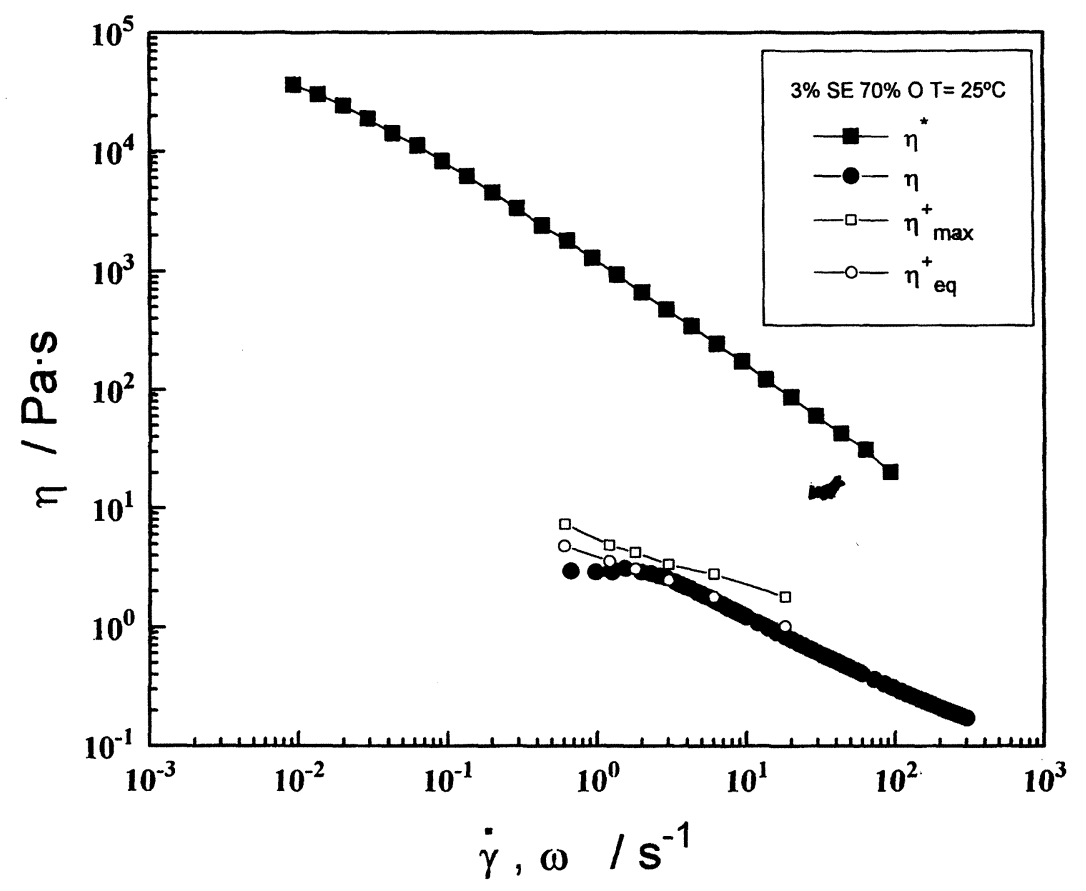

Figura 8

Comparación de los resultados obtenidos en ensayos de cizalla oscilatoria, flujo transitorio y flujo estacionario

\subsubsection{Cantidad de sobreesfuerzo}

El parámetro cantidad de sobre esfuerzo. $\mathrm{S}^{+}$(Ganani y Powell, 1985; Muñoz et al., 1992) da información del crecimiento del esfuerzo que se produce en relación al equilibrio y, por tanto, del descenso del esfuerzo desde el máximo hasta el equilibrio, informando tanto de la destrucción reversible como irreversible.

$$
\mathrm{S}^{+}=\frac{\sigma_{\max }^{+}-\sigma_{\text {eq }}^{+}}{\sigma_{\text {eq }}^{+}}
$$

Los más destacable de estos valores es la variación que se produce con la temperatura como se observa en la figura 9. Un aumento de temperatura produce un descenso de la cantidad de sobreesfuerzo a partir de un determinado valor. De acuerdo con los resultados de viscoelasticidad lineal, a las temperaturas más bajas se produce un mayor grado de estructuración al que contribuyen los puentes de hidrógeno entre los grupos hidroxilo de la sacarosa y el agua. En consecuencia el esfuerzo en el máximo debe ser mayor en dichas condiciones, como se observa en las figuras 1 y 2. También se ha encontrado que la cizalla puede producir deformación y rotura de gotas. A baja temperatura el transporte del tensioactivo hacia la nueva interfase creada se ve dificultado por el aumento de la viscosidad del medio, por lo que el valor de $\sigma^{+}{ }_{\mathrm{eq}}$ tarda más en alcanzarse y los valores de equilibrio serán más bajos de lo esperado, siendo los valores de $S^{+}$ superiores. A alta temperatura el entramado en estado de reposo se encuentra menos estructurado, lo cual conduce a valores, menores de $\sigma^{+}{ }_{\max }$. La redistribución de tamaños de gota está favorecida por la menor viscosidad del medio, por lo que se alcanza el equilibrio con mayor facilidad y como resultado el valor $\mathrm{S}^{+}$es menor.

\subsubsection{Porcentaje de reodestrucción}

Para determinar la destrucción estructural irreversible debida a la cizalla se realizaron ensayos de recuperación estructural tras el cese de la misma, una vez alcanzado el esfuerzo de equilibrio. Los valores de reodestrucción, expresados en tanto por ciento, se han calculado según la expresión (Gallegos et al., 1988):

$$
\% \text { reodestrucción }=100 \frac{\sigma_{\max }^{+}-\sigma_{\text {rec }}^{+}}{\sigma_{\max }^{+}}
$$

donde $\sigma^{+}$rec es el esfuerzo máximo tras la recuperación.

Dicha recuperación está relacionada con la refloculación de las gotas de aceite y por tanto, con la reconstrucción del entramado. La dependencia de la velocidad de recuperación, respecto a la velocidad de cizalla aplicada, es un reflejo de la existencia de enlaces de distinta fuerza entre las gotas que forman los agregados (Sherman, 1967). 


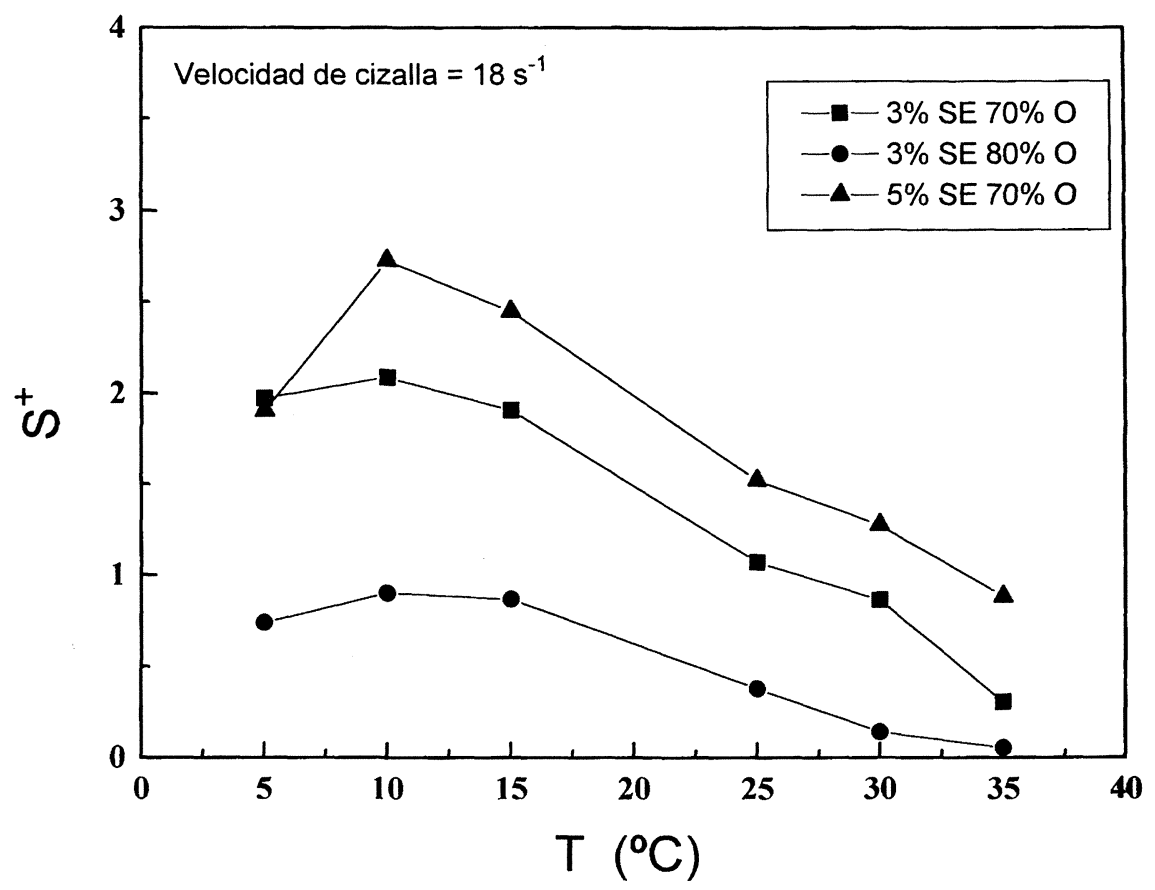

Figura 9

Variación de la cantidad de sobreesfuerzo con la temperatura

Los sistemas más estructurados, es decir, con mayor porcentaje de tensioactivo y de aceite, presentan también un mayor nivel de recuperación (figura 10). La tendencia observada al aumentar la velocidad de cizalla es la de disminuir la recuperación, es decir, la destrucción estructural irreversible tiende a ser más importante. Una redistribución de tamaños producida por la cizalla tenderá, sin embargo, a una recuperación mayor de la esperada, pudiendo incluso producir un descenso del porcentaje de reodestrucción, aunque no de la destrucción irreversible, como sucede para la concentración del $80 \%$ de aceite a alta $\dot{\gamma}$.

\subsection{Cinética de destrucción estructural}

Para estudiar el descenso del esfuerzo o destrucción estructural de la emulsión debida a la cizalla se ha utilizado un modelo cinético formado por una suma de funciones de primer orden (Figoni y Shoemaker, 1983; Shoemaker y Figoni, 1984):

$$
\sigma^{+}-\sigma_{e}^{+}=\sum_{j=1}^{n}\left(\sigma_{o, j}^{+}-\sigma_{e, j}^{+}\right) \exp \left(-k_{j} t\right)
$$

donde $\sigma_{\mathrm{e}}^{+}$es el valor del esfuerzo en el equilibrio y $\sigma_{\mathrm{o}, \mathrm{j}}{ }^{+}, \sigma_{\mathrm{e}, \mathrm{j}}{ }^{+} \mathrm{y} \mathrm{k}_{\mathrm{j}}$ son, respectivamente, el esfuerzo inicial, el esfuerzo en el equilibrio y la constante de velocidad correspondiente al término j-ésimo.

Independientemente de la velocidad y temperatura estudiadas, los valores del descenso del esfuerzo con el tiempo se pueden ajustar, en todos los casos, a una suma de dos exponenciales $(n=2)$, como se puede observar en las figuras 1 y 2 , donde se representan los valores medios obtenidos, siendo el coeficiente de regresión $r>0,99$.

Algunos autores han relacionado las constantes cinéticas, $k_{1}$ y $k_{2}$, con diferentes etapas de la destrucción estructural de una emulsión (Figoni y Shoemaker, 1983; Berjano, 1989). Así, el significado físico del parámetro $k_{1}$, estaría ligado con un proceso de orientación de las unidades esctructurales en la dirección de la cizalla y con el proceso de floculación-defloculación de las gotas emulsionadas. Estos procesos serían perfectamente reversibles. La constante cinética $\mathrm{k}_{2}$, en cambio, podría estar ligada a un proceso reversible y a otro irreversible: el reversible correspondería al proceso de floculación-defloculación mientras que el irreversible podría asociarse a la rotura de ciertos enlaces y a un proceso de coalescencia de las gotas emulsionadas inducida por la cizalla.

No se han encontrado variaciones significativas para $\mathrm{k}_{1}$ con ninguna de las variables estudiadas $(\dot{\gamma}$, $\% \mathrm{SE}, \% \mathrm{O}$ y). Esta circunstancia no es sorprendente si se tiene en cuenta que existe una destrucción estructural muy rápida desde el momento en que se sale de la zona viscoelastica lineal (Guerrero et al., 1996) y, por supuesto, mucho antes de que se alcance el valor del sobreesfuerzo. En definitiva, los valores de $\mathrm{k}_{1}$ deben estar afectados por la inercia del aparato.

La velocidad de cizalla tampoco ejerce influencia significativa sobre la constante cinética $\mathrm{k}_{2}$. Al aumentar la temperatura se produce un ligero aumento de la constante $\mathrm{k}_{2}$. Es decir, la agitación térmica favorece la 


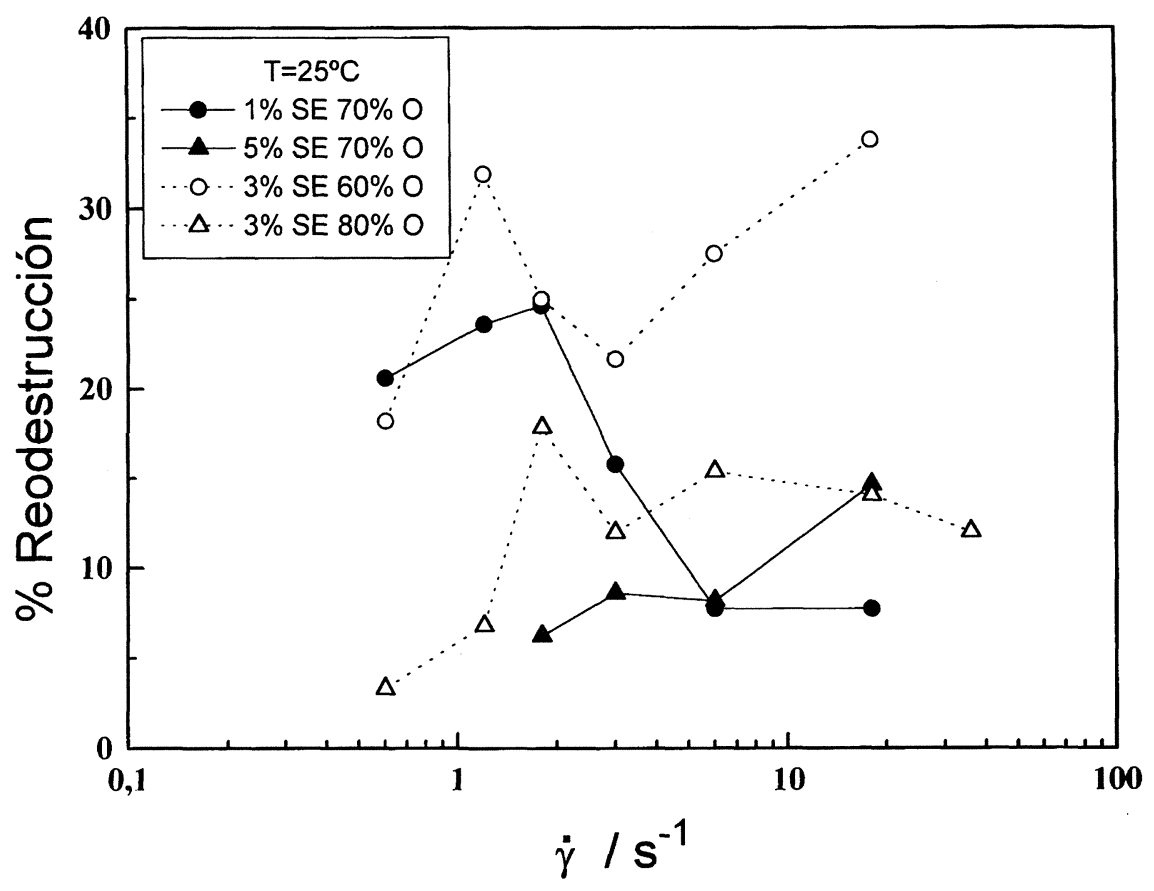

Figura 10

Variación de los valores del porcentaje de reodestrucción a $25^{\circ} \mathrm{C}$ con la velocidad de cizalla

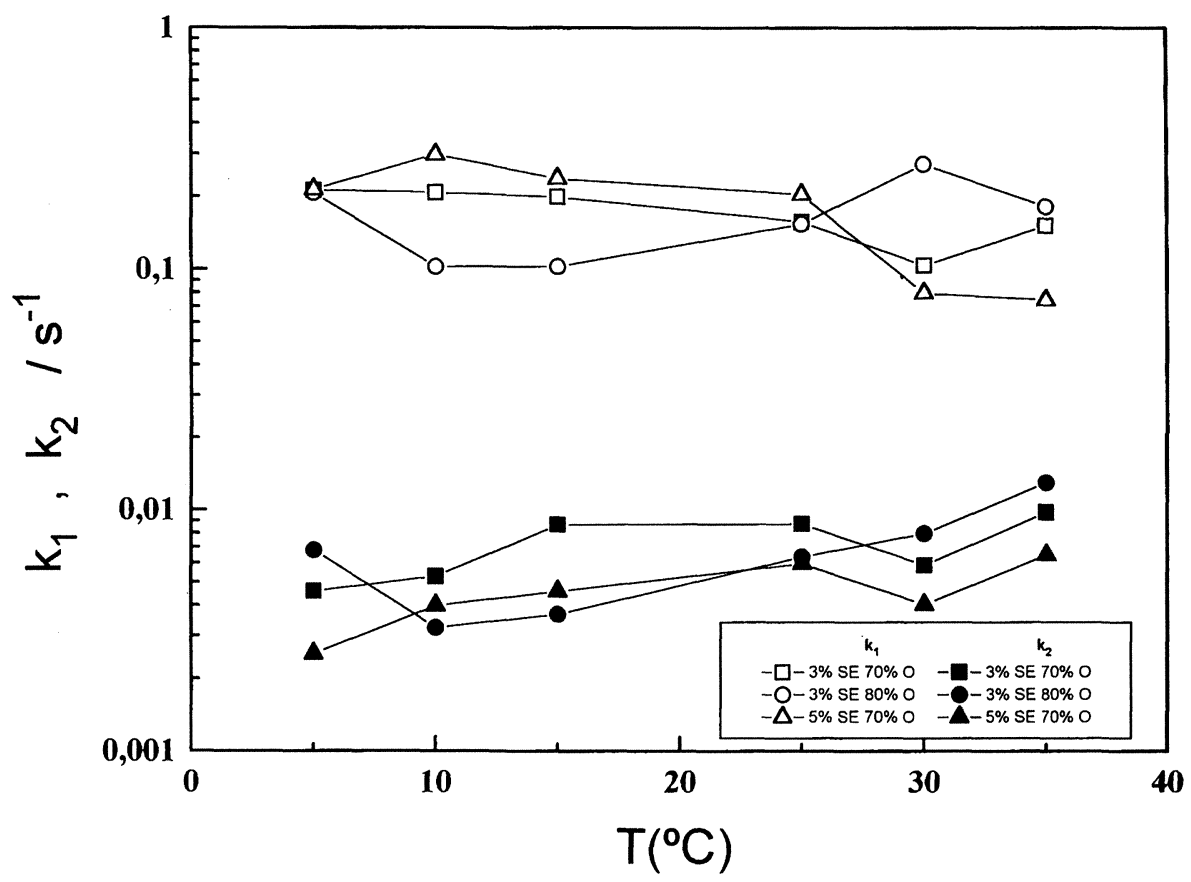

Figura 11

Influencia de la temperatura sobre los parámetros $k_{1}$ y $k_{2}$ del modelo cinético 
cinética de destrucción estructural. Los valores medios de $k_{1}$ y $k_{2}$ se presentan en la figura 11, en función de la temperatura, para diferentes concentraciones de sucroéster y de aceite.

\section{CONCLUSIONES}

Los ensayos de cizalla transitoria dan lugar, en todos los sistemas estudiados, a un máximo del esfuerzo a tiempos muy cortos, seguido de una tendencia a alcanzar un valor de equilibrio, correspondiente al estado estacionario. Sin embargo, bajo condiciones extremas de concentración, temperatura y/o velocidad de cizalla puede observarse un nuevo aumento del esfuerzo. Este aumento del esfuerzo se ha relacionado con una redistribución de tamaños de gota como consecuencia de la cizalla.

Todos los sistemas estudiados presentan un comportamiento pseudoplástico con la velocidad de cizalla a $25^{\circ} \mathrm{C}$, este comportamiento puede ser descrito por el modelo de Oswald-de Waele.

La influencia de la temperatura sobre los sistemas estudiados puede ser descrita por la ecuación de Arrhenius. No obstante, se ha encontrado que la susceptibilidad de la emulsión a la temperatura, relacionada con la energía de activación, es menor a altas temperaturas. Este hecho se puede deber a la redistribución de tamaños de gota que ocurre con la cizalla, la cual está favorecida a alta temperatura.

La cizalla produce una intensa defloculación de los sistemas no cumpliéndose en ningún caso la regla de Cox-Merz. Esta destrucción, que se produce incluso antes de alcanzar el máximo del esfuerzo, presenta un grado de irreversibilidad.

La cinética de destrucción estructural de estos sistemas puede ser descrita en todos los casos por la suma de dos ecuaciones exponenciales. Se ha encontrado que la temperatura favorece la cinética de destrucción estructural.

\section{AGRADECIMIENTOS}

Este trabajo forma parte de un proyecto financiado por la CYCIT (Proyecto ALI90-0503). Los autores desean hacer constar su agradecimiento por dicha financiación.

\section{BIBLIOGRAFÍA}

Akoh, C. C. y Nwosu, C. V. (1992) - -Emulsification properties of polyesters and sucrose ester blends II: Alkyl glycoside polyester".- J. Am. Oil Chem. Soc. 69, 14-19.

Alfaro, M. C. (1993). - "Comportamiento reológico de sistemas emulsionante/agua y emulsiones concentradas o/w que contienen un estearato de sacarosa con un alto grado de poliesterificación»-. Tesis de Licenciatura, Universidad de Sevilla.

Berjano, M., Muñoz, J., Guerrero, A., Flores, V. y Gallegos, C. (1991). - Estudio comparativo de viscosidades obtenidas por ensayos de cizalla dinámica y estacionaria en una mayonesa comercial».- Rev. Agroquím Tecnol. Aliment. 31, 400-410

Berjano, M. (1989). - «Comportamiento viscoelástico no lineal de mayonesas comerciales".- Tesis Doctoral, Universidad de Sevilla.

Cox, W. P. y Merz, E. H. (1958). - "Correlation of dynamic and steady viscosities".- J. Poly. Sci. 28, 619-622.

Cheng, D. C. y Evans, F. (1965). - «Phenomenological characterization of the rheological behaviour of inelastic reversible thixotropic and antithixotropic fluids».- Brit. J. Appl. Phys. 16, 1599-1617.

Darby, R. (1984). - «Emulsion rheology» en "Emulsions and Emulsion Technology».- p. $45-\mathrm{K}$. J. Lissant (Ed).Marcel Dekker, New York.

Desai, N. B. (1990). - «Esters of sucrose and glucose as cosmetics materials".- Cosmetics and Toiletries 105, 99-107.

Dickinson, E. (1994). -«Interactions in protein-stabilized emulsions» en "Progress and Trends in Rehology IV».p. 227. - Gallegos (Ed). - Steinkopff Darmstadt, Germany.

Figoni, P. I. y Shoemaker, C. F. (1983). - «Characterizacion of time-dependent flow properties of mayonnaise under steady shear".- J. Texture Studies 14, 431-442.

Gallegos, C., Berjano, M., García F. P., Muñoz, J. y Flores V. (1988). - «Aplicación de un modelo cinético al estudio del flujo transitorio en mayonesas".- Grasas y Aceites 39, 254-263.

Ganani, E. y Powell, R. L. (1985). - «Transient flow viscosimetry".- J. Rheol, 29, 931-941.

Guerrero, A. F., Gallegos, C. y Ball, H. R. (1992). «Nonlinear viscoelasticity of mayonnaise containing different egg products» en "Theoretical an applied Rheology».p. 718. P. Moldenaers y R. Keunings (ed).- Elsevier Publishers, Amsterdam.

Guerrero, A., Partal, P., Berjano, M., y Gallegos, C. (1996). - "Linear viscoelasticity of $\mathrm{O} / \mathrm{W}$ sucrose palmitate emulsions".- Prog. Colloid Polym. Sci. 100, 246-251.

Madiedo, J. M., Bower C., Mackley, M. R. y Gallegos, C. (1997). - «Reología de emulsiones estabilizadas con biopolímeros".- Grasas y Aceites 48, 405-410.

Muñoz, J., Alfaro, M. C., Cordóbes, F. y Guerrero, A. F. (1994). - «Rheological behaviour of concentrated emulsions containing a sucrose stearate of HLB 5 " en "Progress and Trends in Rheology IV".- p. 195.Gallegos (Ed).- Steinkopff Darmstadt, Germany.

Muñoz, J., Rodríguez, M. y Gallegos C. (1992). -«Transient flow of the lamellar liquid crystal in surfactant/water systems" en "Theoretical and applied Rheology», p. 564. -P. Moldenaers y R. Keunings (Ed).- Elsevier Science Publishers, Amsterdam.

Partal, P., Guerrero, A. Berjano, M., Muñoz, J. y Gallegos, C. (1994). - «Flow behaviour and stability of O/W emulsions stabilized by a sucrose palmitate".- J. Texture Stud. 7 . 331-348.

Rahalkar, R. R. (1992) - - Viscoelastic properties of oil-water emulsions" en "Viscoelastic Properties of Foods".p. 317. -M. A. Rao y J. F. Steffe (Ed).- Elsevier Applied Science, Londres.

Sánchez, M. C. (1997). -Comunicación personal.

Sherman, P. (1967). - «Changes in the rheological properties of emulsions on aging. II. Viscosity changes in water/oil emulsions at rates of shear from $0.133 \mathrm{~s}^{-1}$ to $10.77 \mathrm{~s}^{-1}$ 》. $-J$. Colloid Interface Sci. 24, 97-106.

Shoemaker C. F. y Figoni, P. I. (1984). - «Time-dependent rheological behaviour of foods".- Food Technol. 38, 110-112.

Tadros, T. F. (1993). - «Fundamental principles of emulsion rheology and their applications" en First World Congress on Emulsion».- Vol. 4, p. 237.- EDS (Ed.), París.

Walker, C. E. (1984). - «Food applications of sucrose esters". - Cereal Foods World 29, 286-289.

Yuki, A., Matsuda, K. y Nishimura A. (1990). - «Effect of sucrose polyester on crystalization behaviour of vegetable shortening and margarine fat".- J. Jpn. Oil Chem. Soc. 39, 236-244. 